OPEN

SUBJECT AREAS:

ECOLOGY

EVOLUTIONARY BIOLOGY

HUMAN

SUSTAINABILITY

Received

13 April 2011

Accepted

18 July 2011

Published

5 August 2011

Correspondence and requests for materials should be addressed to O.B. (burger@demogr. mpg.de)

\section{Industrial energy use and the human life history}

\author{
Oskar Burger ${ }^{1}$, John P. DeLong² \& Marcus J. Hamilton ${ }^{3,4}$
}

\begin{abstract}
${ }^{1}$ Max Planck Institute for Demographic Research, Konrad-Zuse-Strasse 1, 18057, Rostock, Germany, ${ }^{2}$ Department of Ecology and Evolutionary Biology, Yale University, New Haven, CT 06520-8106 USA, ${ }^{3}$ Depts. of Biology and Anthropology, University of New Mexico, Albuquerque, NM 87131 USA, ${ }^{4}$ Santa Fe Institute, 1399 Hyde Park Rd, Santa Fe, NM 87501.
\end{abstract}

The demographic rates of most organisms are supported by the consumption of food energy, which is used to produce new biomass and fuel physiological processes. Unlike other species, modern humans use 'extra-metabolic' energy sources acquired independent of physiology, which also influence demographics. We ask whether the amount of extra-metabolic energy added to the energy budget affects demographic and life history traits in a predictable way. Currently it is not known how human demographics respond to energy use, and we characterize this response using an allometric approach. All of the human life history traits we examine are significant functions of per capita energy use across industrialized populations. We find a continuum of traits from those that respond strongly to the amount of extra-metabolic energy used, to those that respond with shallow slopes. We also show that the differences in plasticity across traits can drive the net reproductive rate to below-replacement levels.

nergy and life history traits are connected. Food energy, once processed by digestion, fuels biomass production, enabling growth and reproduction. The energy budgets of most species are constrained primarily by body size because body size sets fundamental limits on how much energy an organism can acquire and physiologically process ${ }^{1,2}$. This in turn sets a fundamental limit on the amount of energy that can be allocated to different components of the life history. Understanding variation in life histories requires understanding how constraints on the use and consumption of energy affect demographic traits. Figure 1 shows the relationship between two measures of biological energy use, basal metabolic rate and field metabolic rate, and body mass, shown with per-capita industrial energy use across several nations for comparison ${ }^{3,4}$. Basal metabolic rate is the minimum energy required to support bodily functions in an inactive state, while field metabolic rate captures the energy demands of active individuals under natural environmental conditions. Both exhibit strong statistical relationships with body mass ${ }^{2-4}$.

Humans in industrialized nations have a very different relationship with energy, one that is independent of body mass. Energy use among the lowest energy-consuming countries is barely above the expected field metabolic rate of a mammal of our size (Figure 1). In contrast, the median level of energy use among industrial populations (i.e., human populations living in contemporary industrialized nations) is about 1,580 watts, indicated by the star in Figure 1, about 10 times greater than expected for a mammal of our size. Individuals living in countries near the top of the energy use spectrum would have basal metabolic rates equivalent to mammals weighing over $92,500 \mathrm{~kg}$, about twice the size of an adult male Sperm Whale (Figure 1). The magnitude and pace of the increase in the human energy budget is likely unprecedented amongst biological species, in part because it occurred without an evolutionary increase to larger body mass.

Extra-metabolic energy can be used for many purposes that change demographic rates in ways that entirely bypass an organism's internal physiology. Most organisms must burn calories derived from food resources in order to lower their mortality rates by, for example, increasing immune function, working harder to stay warm, or avoiding predators. Industrial human environments provide the opportunity to alter mortality rates (and other demographic traits) via the distribution of goods and services that are largely independent of the calories obtained by digesting food. Inputs of extra-metabolic energy can alter demographic patterns both directly, via birth control or medication, for example, or indirectly by improving infrastructure, such as water quality, roads, and schools $\mathrm{s}^{5,6}$. In spite of wide interest in resource constraints on the growth and structure of populations, little is known about the basic statistical responses of human demographic and life history traits to variation in energy use. Understanding such responses is necessary for developing a complete theoretical picture of human demographic processes. 


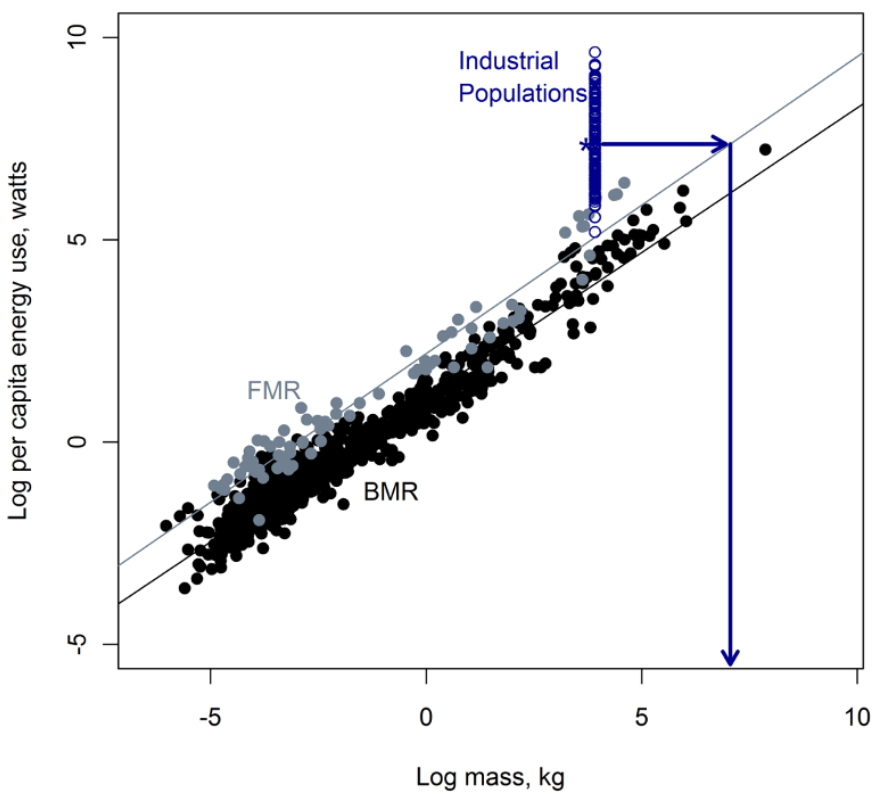

Figure 1 Three kinds of energy use as a function of body mass. Black circles are basal metabolic rate for mammals ${ }^{4}$. Grey circles are field metabolic rate for mammals ${ }^{3}$ The open blue circles are per capita energy use among industrial human populations ${ }^{36}$ with a star at the median value. The arrows illustrate the linking of per-capita energy use to a corresponding mass by way of following the regression between metabolic rate and body size for mammals. Body mass for all human populations is set to $50 \mathrm{~kg}$.

Here we analyze variability in key life history traits across a spectrum of populations that vary widely in per capita energy use. Specifically, we quantify the relationship between human life history traits and extra-metabolic energy use, and ask whether different traits vary in the strength of their response. We take energy as the independent variable in order to test the hypothesis that increases in energy use, independent of body size, influence life history traits consistently, regardless of whether energy is used internally or externally (metabolically or extra-metabolically, respectively). The resulting relationships yield insights into the demography of contemporary human populations, including the perplexing issue of below-replacement fertility rates observed among many wealthy nations today ${ }^{7}$. Furthermore, we show that many of the life history allocation patterns of industrial human populations are simply extensions of the ecological energetics of biological populations.

We investigate human demographic traits using an allometric approach. To introduce this approach we first describe conventional allometry and its role in life history studies, which are generally based on body mass ${ }^{\text {e.g. } 8}$. We convert these relationships from mass to energy. Allometric relationships are power functions of the form $y=c_{0} m^{2}$ where $y$ is some dependent variable of interest that scales with body mass $m$ to the exponent $z$, and $c_{0}$ is a normalization constant. Functions of this form become linear on logarithmic axes, where $z$ is the slope and $\log \left(c_{0}\right)$ the intercept. The value of the slope captures the response of a change in $y$ due to a change in $m$, and when the coefficients are fit to data one says that $y$ scales with mass to the $z$ power.

Essentially all life history traits can be characterized as allometric functions of mass ${ }^{8}$, making body mass perhaps the most fundamental attribute of a species ${ }^{9}$. Biological rates such as birth rate and mortality rate tend to scale negatively with mass as $z$ close to $-1 / 4$, while biological durations such as the time to maturity and the total lifespan tend to scale positively as $z$ about $+1 / 4^{8-11}$. In the following analysis we are concerned primarily with the direction (sign) and steepness of the scaling exponent (slope) $z$, rather than the exact value. Body size is the key constraint on life history traits because, by setting the field metabolic rate, it strongly influences the total amount of energy that can be acquired and allocated among life history traits ${ }^{1}$ (Figure 1). From studies of the role of body size in life history it is clear that there are consistent selective pressures regulating how an organism's size-specific metabolic energy budget influences tradeoffs among life history traits. Figure 1 illustrates how fundamental this size-based constraint is across mammals ${ }^{3,4}$, as well as how different the industrial human energy budget is compared to energy budgets that are constrained primarily by mass. Because of the strong correlation between size and metabolic rate, any trait that scales with body size can also be expressed as a function of metabolic rate. By rewriting allometries in terms of metabolic rate rather than mass, we can examine the change in life history traits in response to changes in energy use. Considering energy instead of mass makes it possible to compare the vital rates of industrial human populations to metabolically constrained species of mammals and primates.

We take a macroscopic approach to assessing how life history traits respond to energy use in order to answer a general question about whether the source of that energy matters. To develop null predictions for the relationships between life history traits and per capita energy use we employ a combination of existing cross-species allometries and the mammal life history model developed in Charnov $^{1,10}$, which predicts the slopes of many life history traits as functions of mass, and after our extension, energy, that are grounded in explicit evolutionary tradeoffs. Given known patterns in crossspecies allometries and Charnov's mammal life history model, the basic null predictions are that 1) rates of mortality, fertility, and population growth should be negatively related to energy use; and 2) the time to a life history event, including to sexual maturity, first reproduction, and lifespan, should be positively related to energy use.

\section{Results}

Birthrate and Juvenile Mortality. Moses and Brown ${ }^{12}$ established the approach of energy-based allometries by demonstrating that birthrate scales with energy use as an extension of the body mass relationships observed across mammals (Figure 2A). They showed that the scaling of birthrate with mass to the $-1 / 4$ power can be reexpressed as a scaling of birthrate with energy use to the $-1 / 3$ power (i.e., $b \propto M^{-1 / 4}$ and $B \propto M^{3 / 4}$ so $b \propto B^{-1 / 3}$ where $b$ is birthrate, $M$ is mass, and $B$ is metabolic rate). The predicted $-1 / 3$ power scaling between birthrate and energy consumption was found not only across biological species but also among industrialized human populations. Further, Moses and Brown (2003) demonstrated the remarkable robustness of this relationship for many different years across countries and over time within the United States between 1850 and the present.

Figure 2B indicates that juvenile mortality, measured here as mortality under age five, is a steeply negative function of energy use with an exponent close to -1 (Table 1). Interestingly, this suggests that rates of juvenile mortality drop proportionally with increasing energy consumption, an exceptionally steep benefit to energy use, as twice the energy consumption leads to half the mortality, which contrasts markedly with adult lifespan (see below).

Adult Lifespan and its Divisions. While birthrate and under five mortality exhibit strong responses to per-capita energy use, adult lifespan exhibits an interesting curvature (Figure 2C). Initially, lifespan rises steeply with per capita energy use and then flattens toward an asymptote at high values ${ }^{13}$. This pattern follows the qualitative prediction in that the slope is positive, but the curvature deviates from the basic log-linear form of an allometric relationship resulting in a slope shallower than the theoretically-predicted $1 / 3$. The observed energy-based allometry for lifespan also differs from the empirical trend across primate species. Primates generally deviate from the overall mammal life history in having slow growth, slow reproduction, and long lifespans ${ }^{14,15}$. But industrial 

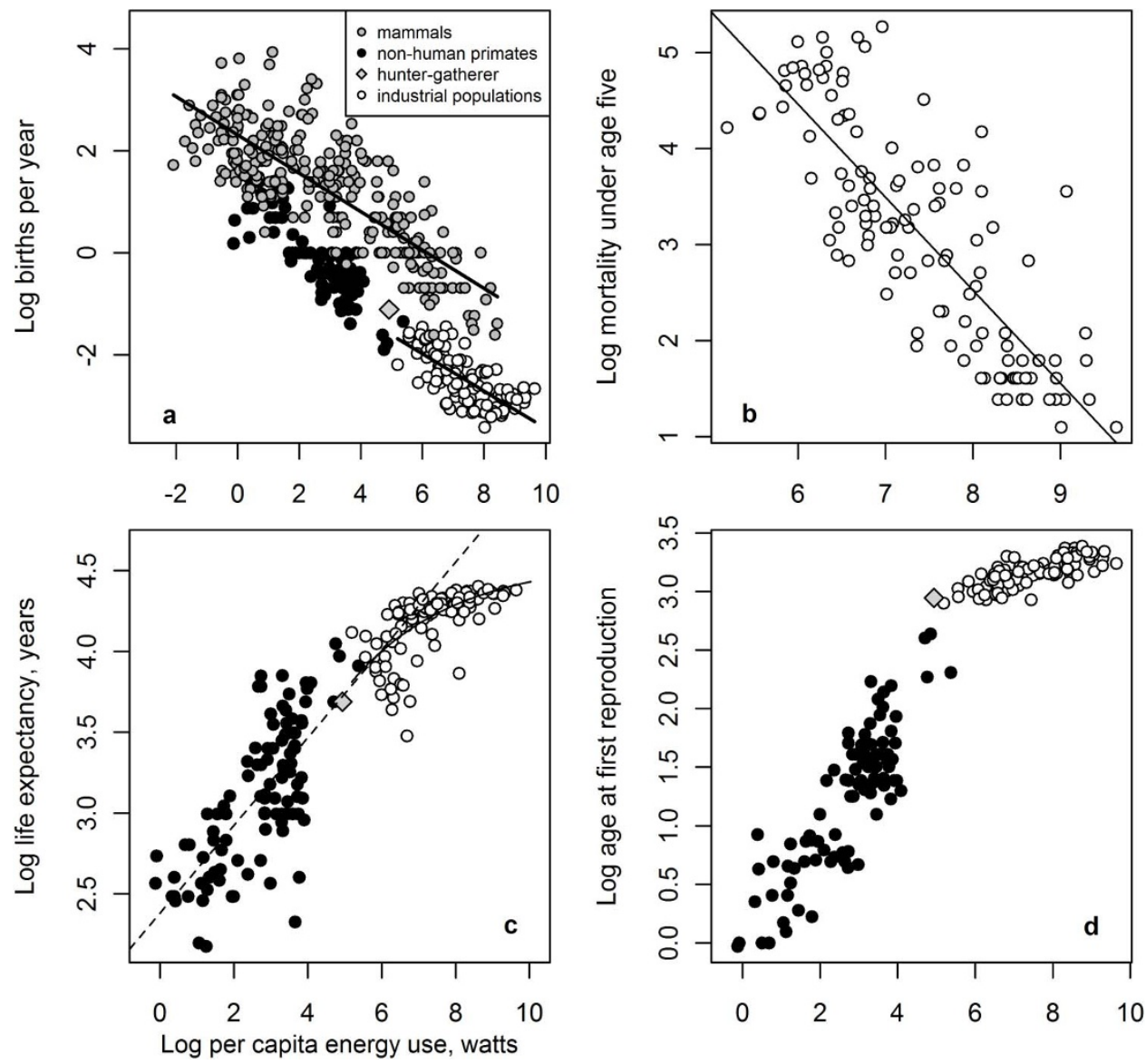

Figure $2 \mid$ Life history traits as functions of per capita energy use. (a) Birthrate. The slopes linking birthrate to per capita energy use are similar for mammals and industrial populations and both are near the predicted value of $-1 / 3^{12}$. (b) Mortality under age five is a steeply negative function of per capita energy use (slope near -1) among industrial nations. (c) Lifespan. Curved solid line is an asymptotic fit to the industrial population data: $\log (\operatorname{lifespan})=4.56+(4.84) * \exp \left(-0.36^{*} \log (\right.$ energy $\left.)\right)$. The dotted line is the linear fit to the nonhuman primate data. Note that the primate data are maximum lifespans whereas the industrial nations are life expectancy at birth. Exactly comparable data for primates would cause the primate data points to shift downward noticeably. (d) Age at first reproduction for industrial nations, nonhuman primates, and an average hunter-gatherer.

human lifespans respond to energy differently than either primates or non-primate mammals. The regression line for the primate data suggests that lifespans among industrial humans are actually shorter than expected based on simply extending the cross-primate allometry to higher levels of energy use. In other words, humans have shorter lifespans than a hypothetical primate species with biological metabolic rates equivalent to the current high levels of per capita energy use (dotted line, Figure 2C).

Age at first reproduction also scales positively, but with a shallower slope than the predicted $+1 / 3$. The scaling of age at first reproduction for humans of 0.1 , although positive as predicted, is shallower than the scaling for primates, 0.45 (Table 1 ).

Figure 3A examines average lifespan alongside some of its major components. All of the pictured life history traits relevant to the divisions of the adult life-course (such as ages at menarche, first birth, and menopause) are significant allometric functions of per capita energy use, but they are not all directionally consistent with the null life history predictions (Table 1). For example, while age at menopause scales positively with energy use, the shallow slope of 0.02 (95\% CI: $0.012-0.038$ ) demonstrates that age at menopause is less responsive to energy consumption than other life history traits. Because the age at first reproduction increases more steeply with energy use than age at menopause, the length of the reproductive lifespan, the time between age at first reproduction and the age at menopause, decreases with energy use (Table 1). As most organisms reproduce for the majority of their adult lives, and lifespan should scale positively with body mass and energy, the negative scaling of reproductive lifespan with energy is contrary to the theoretical expectation and suggests an important constraint on the life history that emerges as a function of increasing energy inputs.

Reproductive delay is the period of time between menarche and age at first reproduction. The relationships in Figure $3 \mathrm{~A}$ indicate that this period of time is an increasing function of energy use among industrial human populations. Plotting reproductive delay across primates to facilitate comparison yields a somewhat surprising result (Figure 3B); reproductive delay increases with body size with an exponent near $1 / 4$ and with metabolic rate to an exponent near $1 / 3$. Interestingly, the slope for reproductive delay as a function of energy use among industrial populations is also near $1 / 3$, almost identical to the primate value (Table 1). While the strength of the relationship is somewhat weak in primates, it is highly significant and the low variance could be attributed to the difficulty of observing the parameters on actual populations. The possibility that reproductive delay may be a general $1 / 4$ power function of body mass across species as well as an underappreciated allometric measure for the pace of the life history warrants further attention.

The Net Reproductive Rate and Below Replacement Growth. Vital rates such as birthrate and mortality do not exist in isolation but are components of aggregate metrics, such as the net reproductive rate. Given the findings above, we may also wonder how the net reproductive rate responds to changes in per capita energy use. 


\begin{tabular}{|c|c|c|c|c|c|}
\hline Dependent Variable & $\begin{array}{l}\text { Intercept } \\
\text { (SE) }\end{array}$ & $\begin{array}{l}\text { Slope } \\
\text { (SE) }\end{array}$ & Adj Rsq & Pvalue & df \\
\hline \multicolumn{6}{|c|}{ industrial populations } \\
\hline Births per year & $\begin{array}{c}0.214 \\
(0.237)\end{array}$ & $\begin{array}{r}-0.365 \\
(0.032)\end{array}$ & 0.522 & 3.67E-21 & 120 \\
\hline Mortality under age five & $\begin{array}{c}10.279 \\
(0.448)\end{array}$ & $\begin{array}{r}-0.969 \\
(0.060)\end{array}$ & 0.684 & 8.56E-32 & 119 \\
\hline Life expectancy* & $\begin{array}{c}3.284 \\
(0.098)\end{array}$ & $\begin{array}{c}0.124 \\
(0.013)\end{array}$ & 0.420 & $3.41 \mathrm{E}-16$ & 121 \\
\hline Age at first reproduction & $\begin{array}{c}2.447 \\
(0.057)\end{array}$ & $\begin{array}{c}0.095 \\
(0.008)\end{array}$ & 0.611 & 2.94E-22 & 99 \\
\hline Age at menopause & $\begin{array}{c}3.705 \\
(0.048)\end{array}$ & $\begin{array}{c}0.025 \\
(0.006)\end{array}$ & 0.413 & 5.67E-04 & 21 \\
\hline Age at menarche & $\begin{array}{c}2.844 \\
(0.053)\end{array}$ & $\begin{array}{c}-0.032 \\
(0.007)\end{array}$ & 0.259 & $1.93 \mathrm{E}-05$ & 58 \\
\hline Reproductive delay & $\begin{array}{c}-0.323 \\
(0.237)\end{array}$ & $\begin{array}{c}0.341 \\
(0.031)\end{array}$ & 0.695 & $3.02 \mathrm{E}-15$ & 52 \\
\hline Net reproductive rate & $\begin{array}{c}2.304 \\
(0.211)\end{array}$ & $\begin{array}{c}-0.302 \\
(0.028)\end{array}$ & 0.515 & 1.37E-18 & 106 \\
\hline Reproductive lifespan** & $\begin{array}{c}35.760 \\
(3.521) \\
\text { nonhuman }\end{array}$ & $\begin{array}{r}-1.359 \\
(0.450) \\
\text { primates }\end{array}$ & 0.300 & 7.34E-03 & 18 \\
\hline Maximum lifespan & $\begin{array}{c}2.380 \\
(0.079)\end{array}$ & $\begin{array}{c}0.271 \\
(0.027)\end{array}$ & 0.507 & 6.07E-17 & 98 \\
\hline Age at first reproduction & $\begin{array}{c}0.035 \\
(0.082)\end{array}$ & $\begin{array}{c}0.445 \\
(0.028)\end{array}$ & 0.730 & 5.49E-29 & 95 \\
\hline Reproductive delay & $\begin{array}{r}-1.338 \\
(0.285)\end{array}$ & $\begin{array}{c}0.321 \\
(0.094)\end{array}$ & 0.159 & $1.13 \mathrm{E}-03$ & 56 \\
\hline Age at menarche & $\begin{array}{r}-0.306 \\
(0.112)\end{array}$ & $\begin{array}{c}0.495 \\
(0.037)\end{array}$ & 0.685 & 9.38E-22 & 79 \\
\hline Reproductive lifespan** & $\begin{array}{c}7.980 \\
(2.184)\end{array}$ & $\begin{array}{c}4.600 \\
(0.724)\end{array}$ & 0.319 & $1.05 \mathrm{E}-08$ & 83 \\
\hline \multicolumn{6}{|c|}{ mammals, excluding primates } \\
\hline Births per year & $\begin{array}{c}2.312 \\
(0.064)\end{array}$ & $\begin{array}{c}-0.376 \\
(0.016)\end{array}$ & 0.662 & $1.08 \mathrm{E}-67$ & 278 \\
\hline
\end{tabular}

The net reproductive rate, $R_{0}$, combines birthrate and survival (lifespan) into a single quantity in units of daughters per mother ${ }^{16}$. Non-growing populations have, by definition, lifetime reproductive rates such that each mother exactly replaces herself; i.e., $R_{0}=1$ at replacement. If $R_{0}$ is greater than 1 a population is growing and if less than 1 it is declining.

Across industrial populations $R_{0}$ drops with per capita energy use (Figure $3 \mathrm{C}$ ), consistent with the commonly noted trend for mortality and fertility to be lower among higher energy consuming nations or social strata ${ }^{17,18}$. Figure $3 \mathrm{C}$ shows that $R_{0}$ declines with per capita energy use with a slope near $-1 / 3$, and that 51 of the 107 (48\%) nations represented are at or below replacement levels of growth in terms of $R_{0}$. If these were biological populations in the wild, we would predict that these populations were headed toward extinction (ignoring immigration). Because the component parts of $R_{0}$ are energy-based allometries, our results shed light on why net reproductive rates frequently drop to such exceptionally low levels in human populations.

$R_{0}$ is the product of birthrate $b$, lifespan $E$, and the probability of survival to reproductive age $S$, so $R_{0}=S * b * E^{10}$. It is well known that $b$ and $E$ are allometric functions of mass $M, E=a_{0} M^{1 / 4}, b=b_{0} M^{-1 / 48}$, where $a_{0}$ and $b_{0}$ are constants. Figures $2 \mathrm{~A}$ and $2 \mathrm{C}$ demonstrate that they are functions of energy as well. As a result, $R_{0}$ can be written as a function of mass:

$$
R_{0}=S *\left(a_{1} M^{1 / 4} * a_{2} M^{-1 / 4}\right),
$$

Or equivalently, as a function of energy:

$$
R_{0}=S *\left(a_{3} B^{1 / 3} * a_{4} B^{-1 / 3}\right),
$$

where $B$ is either metabolic rate, or extra-metabolic energy use $e^{12,19}$. In equation 1 , the exponents of $1 / 4$ for lifespan and $-1 / 4$ for birthrate cancel, and so $R_{0}$ must equal one for any non-growing population regardless of mass. However, equation 2 provides a means of evaluating the effects of per capita energy use on $R_{0}$ via the energy-based allometries for $b$ and $E$, by writing it generally as a function of energy with unknown coefficients:

$$
R_{0}=S *\left(a_{3} B^{z_{1}} * a_{4} B^{z_{2}}\right),
$$

Because the balance between birth and mortality (inverse lifespan) drives changes in $R_{0}$ we define $\delta=a_{3} * a_{4}$, and $z=z_{1} * z_{2}$. Taking the logarithm of both sides yields

$$
\log \left(R_{0}\right)=\log (S)+\log \left(\delta B^{z}\right),
$$

where the balance between fertility and mortality is captured by the value of $z$. Otherwise typical biological populations will have $z=0$ because the empirical relationships between $B$ and the vital rates would cancel each other out, as in equations 1 and 2 . If $S$ is held constant in equation 4 then it is apparent that if either $b$ or $E$ scaled differently than predicted (i.e., other than $+/-1 / 3$ ) across populations, then $R_{0}$ would vary with energy use. This means that $z$ captures the balance between reproduction and lifespan (avoiding mortality) and demonstrates how population growth rates might be affected by varying allometric constraints on $b$ and $E$. Holding $S$ constant here is quite reasonable because $S$ varies only slightly as a function of per capita energy use for high values, those above the median, and in fact varies relatively little across the entire dataset (Figure 3D). As such, juvenile survival varies little among wealthier nations, which are generally those that have either gone through, or are going through the demographic transition to below-replacement fertility.

To investigate the effect of $z$ on $R_{0}$ we examine the derivative of $\log \left(R_{0}\right)$ with respect to $B$ (or $\left.M\right)$ :

$$
\frac{d \log R_{0}}{d B}=\frac{z}{B(\text { or } M)}
$$

or as a function of $\log (B)$ :

$$
\frac{d \log R_{0}}{d \log B}=z
$$

When $z$ is negative the net reproductive rate declines, and any species with a negative $z$ should have fewer offspring per reproductive lifetime. The relationships in Figures $2 \mathrm{~A}$ and $2 \mathrm{C}$ can be used to test this prediction. A linear fit to the lifespan relationship in Figure 2C gives a slope of $\sim 0.12$, which can be combined with the slope for birthrate to give $\frac{d \log R_{0}}{d \log B}=z=-0.365+0.124=-0.241$, which is just within the $95 \%$ confidence intervals for slope of $\log (\mathrm{R})$ vs. $\log (\mathrm{B})$ in Figure 3C (95\% CI: -0.358 to -0.246$)$, but this calculation underestimates $z$ because it does not account for the curvature in the relationship between lifespan and per-capita energy use. If we subset the data to values of energy use above the asymptote in the lifespan data the slope falls more squarely within intervals. Nonetheless, it is clear that lifespan responds to energy-based allometric constraints differently and less plastically than does birthrate. Thus the imbalance between the energetic dependence of birth and death rates may be related to the drop in net reproductive rate to below replacement levels.

\section{Discussion}

Our results show that many human life history traits can be described as allometric functions of per capita energy use. In some cases these allometries are broadly consistent with the variation in metabolic rates across species, but in others the relationship differs in direction or shape. The ability to harvest large sums of extra-metabolic energy from the environment raises the total per capita energy budget to levels far beyond the field metabolic rate predicted for a primate of 

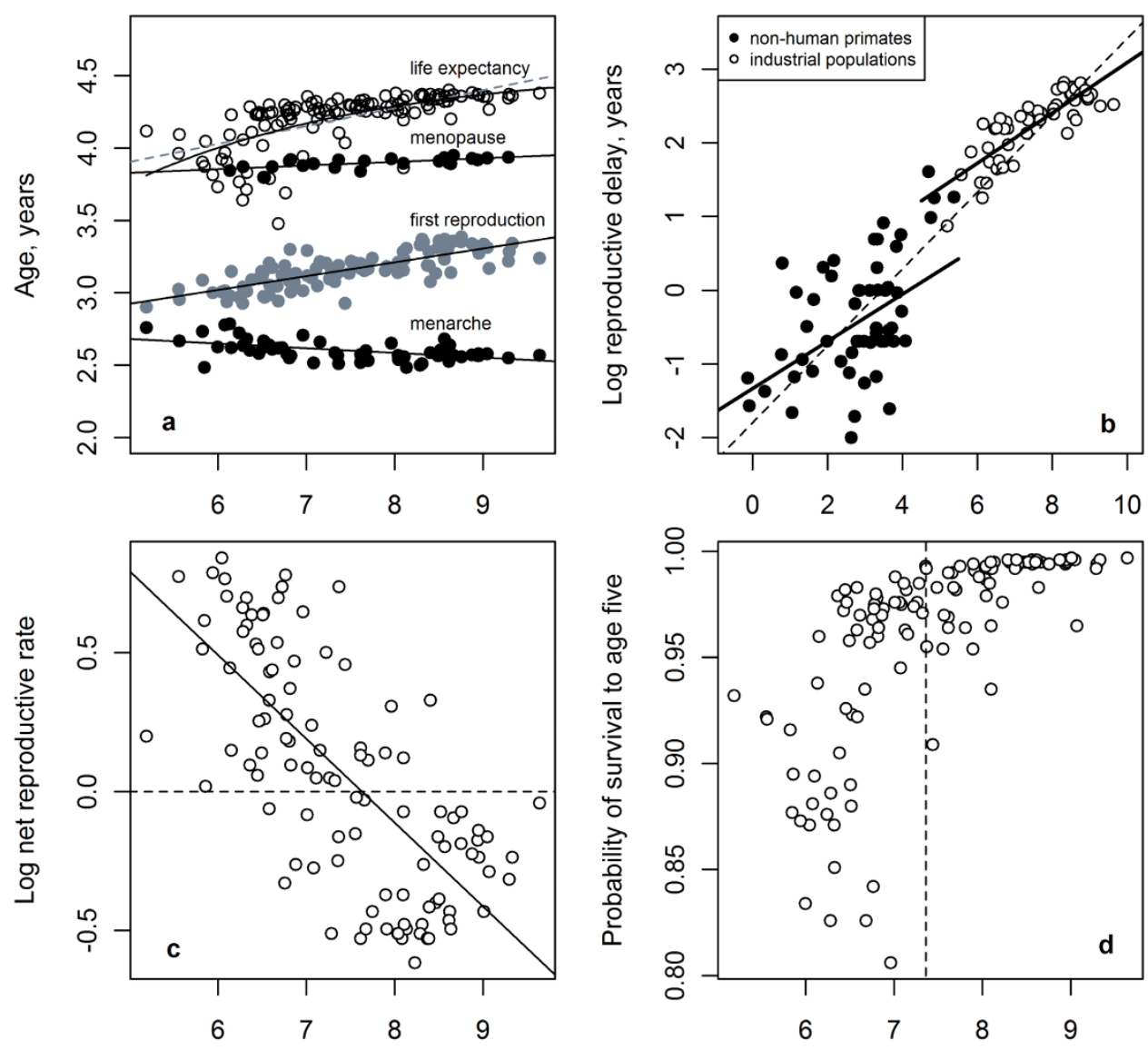

Figure $3 \mid$ Life history traits as functions of per capita energy use. (a) The lifespan and its major divisions for industrial populations (nations).

(b) Reproductive delay (age at menarche - age at first reproduction) varies similarly with per capita energy use among nonhuman primates and industrial human populations. The dashed line is a linear fit to the combined datasets, nonhuman primates and industrial nations. (c) The net reproductive rate for industrial populations. Horizontal dotted line drawn at $\log \left(\mathrm{R}_{0}\right)=0$. Growing populations are above the line and declining populations are below it (not accounting for migration or other dynamics). (d) Probability of survival to age five for industrial populations. Median value of energy use indicated with the dashed line.

our size (Figure 1). This increase changes the constraints on human life history traits. Some life history traits respond to energy with steeper allometric exponents than others (Table 1), which indicates that some traits respond more readily to extra-metabolic investments of infrastructure, goods, and services, while others are more closely tied to physiological constraints and hence are not as easily adjusted by technological development. The differences in response, and their underlying constraints, can drive high-energy populations to belowreplacement levels of net reproductive rate, which in turn suggests a route to a novel energy-based explanation for the phenomenon widely referred to as the demographic transition ${ }^{17,20}$.

The traits that are consistent with the null predictions, in that they scale with energy with the predicted sign (direction), are birthrate, juvenile mortality, reproductive delay, age at first reproduction, and age at menopause. Traits that respond in the opposite directions, and thus run contrary to a life history or allometric expectation, are the length of the reproductive lifespan and the age at menarche (Table 1). Birthrate is predicted especially well, quantitatively matching the slope for mammalian patterns ${ }^{12}$, adding to the idea that processes of industrial development, like urbanization, may have particularly consistent influences on offspring investment tradeoffs ${ }^{21}$. Lifespan increases, which is consistent with the direction of the cross-species pattern, but differs in shape from the standard log-linear allometries by bending toward an asymptote at high levels of per capita energy use $\mathrm{e}^{13}$.

These traits can also be described as varying along a continuum with respect to how steeply each responds to per capita energy use
(Table 1). Juvenile mortality is the most plastic, followed by birthrate, reproductive delay, age at first reproduction, lifespan, and age at menopause is the least plastic. Not surprisingly, early life mortality and late life mortality are on opposite ends of this spectrum; mortality at young ages can be more efficiently reduced than mortality at late ages. Age at menopause and life expectancy exhibit the leastplastic responses to energy use of any of the traits examined. Both of these may be more closely tied to physiological processes than the traits that exhibit highly plastic (steep) responses to changes in energy use. For example, extra-metabolic energy can have a very strong effect on lowering infant mortality but menopause responds with a very shallow slope, perhaps reflecting the hard physiological constraint of follicle depletion that leads to the onset of menopause ${ }^{22}$. Menopause and lifespan are also influenced by extra-metabolic energy, but their shallower slopes demonstrate weaker responses, and likewise imply that biology and actual physiological metabolism constrain these traits more than others. Moreover, this suite of statistical responses implies that the drivers of these patterns are closely related to the energetic properties of human environments, primarily extrinsic variables, and not uniquely based on social or cultural phenomena that might be posited to exist independent of energy use.

Physiological constraints are the most likely cause of the asymptotic relationship between lifespan and energy use. While average lifespans can be extended using extra-metabolic energy (by improved sanitation and increased access to healthcare, for example), the biophysical requirement of having to metabolize energy for somatic 
maintenance sets a constraint that cannot be indefinitely stretched by inputs of extra-metabolic energy. Thus far, however, human populations have continued to lower mortality in all age groups and the limits of this trend have been notably difficult to estimate ${ }^{23,24}$. Nonetheless, while industrialized humans are exceptionally longlived for mammals ${ }^{23}$, they are not as long-lived as predicted for a primate with an equivalent body size given our extra-metabolic energy consumption. That is, a hypothetical primate species following primate allometric patterns but with the metabolic rate equivalent to the energy use of a wealthy industrial population would be very large and have a very slow birth rate, but would also produce offspring for longer periods of time, keeping the net reproductive rate near replacement. Without the allometrically appropriate mass and physiology, however, contemporary human life histories face novel constraints that can drive the net reproductive rate to below replacement levels.

The actual length of the reproductive period decreases with increasing energy use, implying that the factors accompanying increased energy consumption may increase, rather than decrease, constraints on the length of the reproductive lifespan. For example, Korperlainen $^{20}$ showed that reproductive periods reduced to very short intervals (less than five years) accompanying socio-economic development in Finland between 1870 and 1949. A more constrained, shortened, reproductive lifespan must affect tradeoffs between reproduction and investments in the accumulation of wealth and or/or status, or "maintenance costs" in life history terms, because the amount of time during which offspring can be produced directly constrains the number that can be produced in a lifetime ${ }^{25}$. Therefore, the shortening of the reproductive lifespan is likely an important part of the story leading to below replacement reproduction. While not specifically examined, Figure $3 \mathrm{~A}$ also suggests that the maximum potential reproductive lifespan is increasing with energy use as the time between menarche and age at menopause seems to increase. It seems that maintaining a high-quality industrial niche requires that increasingly large fractions of the potential reproductive period be converted to forms of growth and maintenance (economic productivity) that compete with or constrain maximum potential reproductive output ${ }^{21,26}$.

While human energy budgets have increased dramatically with the advent of the industrial age, it is not necessarily the case that subsidizing the life history budget with additional resources occurs only in industrial contexts. Many anthropologists have noted that the evolved human life history pattern consists of costly and dependent offspring. Among hunter-gatherers it is clear that to raise an offspring from weaning to adulthood requires substantial subsidies from the foraging efforts of individuals other than the parents, including unrelated males, older siblings, and grandmothers ${ }^{27-30}$. Indeed, a more remarkable feature of the evolved human life history pattern is that the relative cost of offspring seems to assume the presence of a social network that will increase food supplies for the offspring beyond that which the parents would be capable of supplying on their own ${ }^{27,28}$. As such, the contemporary industrial energy budget should be seen as the most recent manifestation of an evolving human energy budget, an amplification of a pattern that can be traced back to our hunter-gatherer roots. In this light the industrial energy budget could be seen as increasing the energy budget by large quantities, but it would not be strictly novel to industrial peoples (although the form of the energy is of course quite different). If so, the fact that some scaling patterns are preserved across such complex fuel sources and distribution networks is especially interesting and implies some emergent structure and/or deep evolutionary roots to how human societies allocate resources.

A note about causality. Our analysis begins with a factual premise. Humans have increased their energy budgets with the addition of energy from industrial sources. As with all organisms, our physiology and life histories evolved with an energy budget set primarily by body size. We find that demographic traits respond to this extra-metabolic energy in a variety of ways. We do not investigate the various possible proximate mechanisms for each trait we consider. There are numerous and varied routes by which energy may link goods, services, and infrastructure to health, demographic rates, and economic investment strategies. However, a necessary step preceding the explanation of any macroecological pattern is its empirical recognition ${ }^{31}$. We have introduced several statistically significant allometric relationships that beg explanation, and our central objective was simply to ask whether such patterns exist and to identify their basic characteristics. Our analysis highlights the need to understand the ways that energy can limit the mechanisms generating these relationships. As energy is ultimately responsible for all biochemical work, it must play a causal role in the processes linking societal infrastructure to the growth and maintenance of the humans depending on the resources these infrastructures provide.

In sum, all of the life history traits we examined are significant functions of per capita energy use. Many of them vary in ways similar to the variation of metabolic rate across species. The balance of these traits provides important insights for the relationship of human demographics to the exceptionally large industrial energy supply. The patterns examined here take initial steps toward understanding how extra-metabolic energy use impacts human life history traits. While humans have a life history mediated through technology, energy, and myriad social factors, we demonstrate that there is a consistent structure to the demographic patterns of industrialized populations, at least at the large aggregated scales examined here. On the one hand, given the flexibility and rapid changes of technology and energy, combined with the complex social interactions of modern societies, it may seem surprising that allometric relationships describe the life history traits of industrial humans at all. On the other hand, given that energy is fundamental to all ecological and evolutionary processes, including population dynamics and life histories, perhaps we should not be surprised to find that it affects human demographics as well.

\section{Methods}

Here we present the sources and relevant calculations for the variables depicted in the Figures. Basal and field metabolic rates (BMR and FMR) for mammals were obtained from the two most recent compilations we are aware of ${ }^{4,32}$. BMR was originally reported in units of milliliters of oxygen consumption per hour and FMR in units of kilojoules per day, each of which we converted into watts (joules per second). Per capita energy use for industrial populations is from the Earthtrends database at the World Resource Institute (WRI). The WRI provides methodological details, the most germane of which we repeat here: "Total energy consumption per capita measures the amount of primary energy consumed, on average, by each person living in a particular country or region for the year indicated. All primary sources of energy, including coal and coal products, oil and petroleum products, natural gas, nuclear, hydroelectric, etc., are included here. Data are reported in kilograms of oil equivalent (kgoe) per person. Consumption equals indigenous production + imports - exports - energy delivered to international marine bunkers $+/-$ stock changes...Energy losses from transportation, friction, heat, and other inefficiencies are included in these totals." These data were also converted into Watts ${ }^{12}$.

A limitation of this measure of energy use is that we assume that the average level of energy consumption in an aggregated and coarse dataset is a proxy for variation in individual-level access to energy across countries. Within-country variation in access to energy is not included and this has to be an important source of variation in the patterns we depict here. An open and interesting question is the degree to which these patterns would be similar across social strata within countries given the asymmetry in the distribution of individual access to energy sources.

To derive allometric relationships linking body mass to metabolic rate in Watts for mammals and primates we use empirical fits to the data $\mathrm{in}^{3} \mathrm{and}^{4}$. The final estimates were: $\mathrm{FMR}=8.89 *$ Mass $^{0.734}$ for mammals and FMR $=7.56 *$ Mass $^{0.765}$ for primates (mass in $\mathrm{kg}$ ). The primate intercept had to be estimated based on the difference between the heights of the functions for FMR and BMR in mammals (because of the strongly parallel nature of the fits of BMR and FMR to mass, Figure 1).

All non-human primate data are from ${ }^{33}$. Birthrate and body size data for mammals are from ${ }^{12}$; for hunter-gatherers from ${ }^{27}$; and for industrial populations from WRI. Annual birthrate for industrial populations is the total fertility rate divided by the difference of the best fit allometric functions between age at menopause and age at first reproduction vs. per capita energy use, with the difference between the first reproduction and menopause taken as the length of the reproductive lifespan. Moses 
and Brown use an average reproductive lifespan of 20 years and get the same basic result ${ }^{12}$. Mortality under age five and life expectancy data for industrial populations are from WRI. Because mortality at ages under five is available in units of deaths per 1000 , the corresponding probability of survival was calculated as (1000-number of deaths under age five)/1000 (Figure 3C). Age at first reproduction for industrial populations were obtained as Selected Indicators from the United Nations Population Division $^{34}$. Ages at menarche and menopause for industrial humans are from ${ }^{35}$. The reproductive lifespan was crudely estimated as the time between first birth and menopause in human populations and the time between first birth and lifespan in nonhuman primates. Both are certainly overestimates and the values in Table 1 should be seen as only rough approximations for this variable. For the reproductive delay regression we excluded negative values. Net reproductive rate data are national averages from 2000 to 2005 and were obtained from the UN website ${ }^{34}$.

All variables were natural-log-transformed before analysis, except for length of reproductive lifespan, and best-fit lines obtained by ordinary least squares regression. The asymptotic fit applied to the lifespan data was implemented in $\mathrm{R}$ version 2.9.1. Outliers are removed from the industrial populations (all of which are oil producing nations), but including them does not appreciably alter the results and their relevance is discussed in Moses and Brown ${ }^{12}$ and DeLong et al. ${ }^{19}$.

1. Charnov, E. L. Evolution of Life History Variation among Female Mammals. Proc. Natl. Acad. Sci. USA 88, 1134-1137 (1991).

2. Brown, J. H., Gillooly, J. F., Allen, A. P., Savage, V. M. \& West, G. B. Toward a metabolic theory of ecology. Ecology 85, 1771-1789 (2004).

3. Nagy, K. A. Field metabolic rate and body size. J. Exp. Biol. 208, 1621-1625 (2005)

4. Sieg, A. E. et al. Mammalian metabolic allometry: do intraspecific variation, phylogeny, and regression models matter? Am. Nat. 174, 720-733 (2009).

5. Smil, V. Energy in the Twentieth Century: Resources, Conversions, Costs, Uses, and Consequences. Ann. Rev. Energy and Env. 25, 21-51 (2000).

6. Brown, J. H. et al. Energetic Limits to Economic Growth. BioScience 61, 19-26 (2011).

7. Kohler, H. P., Billari, F. C. \& Ortega, J. A. The Emergence of Lowest-Low Fertility in Europe During the 1990s. Pop. Dev. Rev. 28, 641-680 (2002).

8. Calder, W. A. Size, Function, and Life History. (Dover Publications: Mineola 1984)

9. Peters, R. H. The Ecological Implications of Body Size. (Cambridge University Press: Cambridge, 1983).

10. Charnov, E. L. Life History Invariants. (Oxford University Press: New York, 1993).

11. Savage, V. M. et al. The predominance of quarter-power scaling in biology. Funct. Ecol. 18, 257-282 (2004).

12. Moses, M. E. \& Brown, J. H. Allometry of human fertility and energy use. Ecol. Lett. 6, 295-300 (2003).

13. Preston, $\mathrm{S}$. H. The changing relation between mortality and level of economic development. Int. J. Epidem. 36, 484 (2007).

14. Charnov, E. L. \& Berrigan, D. Why do Female Primates Have Such Long Lifespans and So Few Babies? or Life in the Slow Lane. Ev. Anth. 1, 191-194 (1993).

15. Hawkes, K. Slow Life Histories and Human Evolution. The Evolution of Human Life History 95-126 (2006).

16. Preston, S. H., Heuveline, P. \& Guillot, M. Demography: measuring and modeling population processes. Pop. Dev. Rev. 27, 365 (2001).

17. Borgerhoff Mulder, M. The demographic transition: are we any closer to an evolutionary explanation? TREE 13, 266-270 (1998).

18. Lee, R. The demographic transition: three centuries of fundamental change. J. Econ. Per. 17, 167-190 (2003).

19. DeLong, J. P., Burger, O. \& Hamilton, M. J. Current Demographics Suggest Future Energy Supplies Will Be Inadequate to Slow Human Population Growth. PLoS ONE 5, e13206 (2010).
20. Korpelainen, H. Human life histories and the demographic transition: A case study from Finland, 1870-1949. Am. J. Phys. Anth. 120, 384-390 (2003).

21. Mace, R. Reproducing in cities. Science 319, 764 (2008).

22. Gosden, R. G. \& Telfer, E. Numbers of follicles and oocytes in mammalian ovaries and their allometric relationships. J. Zoology 211, 169-175 (1987).

23. Oeppen, J. \& Vaupel, J. W. Broken limits to life expectancy. Science 296, 1029 (2002).

24. Tuljapurkar, S., Li, N. \& Boe, C. A universal pattern of mortality decline in the G7 countries. Nature 405, 789-792 (2000).

25. Kaplan, H. A Theory of Fertility and Parental Investment in Traditional and Modern Human Societies. Yearbook Phys. Anth. 39, 91-135 (1996).

26. Kaplan, H., Lancaster, J. B., Tucker, W. T. \& Anderson, K. G. Evolutionary approach to below replacement fertility. Am. J. Hum. Biol. 14, 233-256 (2002).

27. Burger, O., Walker, R. \& Hamilton, M. J. Lifetime reproductive effort in humans. Proc. R. Soc.B 277, 773-777 (2010).

28. Hrdy, S. B. Evolutionary Context of Human Development: The Cooperative Breeding Model. Attachment and Bonding: A New Synthesis 9-32 (2006).

29. Reiches, M. W. et al. Pooled energy budget and human life history. Am. J. Hum. Biol. 21, 421-429 (2009).

30. Hill, K. \& Hurtado, A. M. Cooperative breeding in South American huntergatherers. Proc. R. Soc. B 276, 3863 (2009).

31. Brown, J. H. Macroecology. (University of Chicago Press: Chicago, 1995).

32. Nagy, K. A., Girard, I. A. \& Brown, T. K. Energetics of free-ranging mammals, reptiles, and birds. Ann. Rev. Nut. 19, 247-277 (1999).

33. Lindenfors, P. Sexually antagonistic selection on primate size. J. Ev. Biol. 15, 595607 (2002).

34. United Nations Population Division United Nations Population Division Home Page. (2010).at <http://www.un.org/esa/population/>

35. Thomas, F., Renaud, F., Benefice, E., De Meeüs, T. \& Guegan, J. F. International variability of ages at menarche and menopause: patterns and main determinants. Hum. Biol. 73, 271-290 (2001).

36. World Resource Institute World Resource Institute, EarthTrends. (2010).at $<$ http://earthtrends.wri.org/searchable_db/index.php?theme $=6>$

\section{Acknowledgments}

This manuscript benefitted from guidance and/or criticism from Melanie Moses, James $\mathrm{H}$. Brown, James Boone, Robert Walker, Daniel Levitis, William Burnside, Rachel Margolis, Dan Wieczynski, and two anonymous reviewers. OB thanks the Max Planck International Research Network on Ageing (MaxNetAging) and the Max Planck Institute for Demographic Research; JPD thanks Yale University and David Vasseur for support; MJH thanks NSF and the Rockefeller Foundation.

\section{Author contributions}

The production of this paper was a group effort. $\mathrm{OB}$ conceived of the research, compiled and analyzed the data, and was the primary writer, but all authors contributed to all aspects of manuscript production.

\section{Additional information}

Competing financial interests: The authors declare no competing financial interests.

License: This work is licensed under a Creative Commons

Attribution-NonCommercial-ShareAlike 3.0 Unported License. To view a copy of this license, visit http://creativecommons.org/licenses/by-nc-sa/3.0/

How to cite this article: Burger, O., DeLong, J.P. \& Hamilton, M.J. Industrial energy use and the human life history. Sci. Rep. 1, 56; DOI:10.1038/srep00056 (2011). 